\title{
Covariance Structure Analysis of a Model for Use in Administrative Evaluation Regarding End-Of-Life Care in Nursing Homes - Promotion of the PDCA Cycle
}

\section{Omura $\mathbf{M}^{*}$}

School of Nursing, Seirei Christpher University, Japan

*Corresponding author: Mitsuyo Omura, Seirei Christpher University, 3453 Mikatahara, Kitaku, Hamamatsu, Shizuoka, 433-8558, Tel: 0534391493; Email: mitsuyo-o@seirei.ac.jp
Research Article

Volume 4 Issue 2

Received Date: March 09, 2020

Published Date: April 17, 2020

DOI: $10.23880 /$ nhij-16000219

\section{Abstract}

The purpose of this study was to verify the relationships linking implementation of the PDCA cycle for end-of-life care in nursing homes, nursing ability and the results of end-of-life care based on a model for administrative evaluation of nursing care. A further aim was to identify factors toward the improvement of practical nursing skills. A total of 256 administrators of nursing homes registered as National Council for Elderly Welfare Facilities were invited to take part in the study's selfreported questionnaire survey, the evaluation model was verified using structural equation modeling. The factors identified for practical nursing skill improvement were 1) consideration of easily understandable explanations, guidelines and plans for end-of-life care in nursing homes, and securement of agreement from residents and their families, and 2) regularization of meetings with staff and families for reflection after a resident passes away. These factors are expected to promote the PDCA cycle for end-of-life care in nursing homes.

Keywords: Nursing home; End-of-life care; Nursing administrator; Evaluation model; Nursing ability; Covariance Structure Analysis

\section{Introduction}

While local inclusion care systems have been promoted in our country, nursing homes, which are regarded as bases of community-based welfare, are required to assume a role of home for end-of-life care (hereinafter called EoLC) even more. The government presented the policy for raising the additional aid for EoLC from 2015 and reinforcement of the EoLC system as an attempt to focus on EoLC at nursing homes [1]. Moreover, the government is calling for promotion of the PDCA cycle of EoLC in which new measures that strengthen the past EoLC systems were included. This PDCA cycle is a comprehensive system aiming at maintenance of a system needed for practicing EoLC and improvement of care quality. This cycle consists of P (Plan) as "maintenance of the system", D (Do) as "EoLC", C (Check) as "reflection" and
A (Action) as "improvement of the system". Satisfactory maintenance of this cycle would allow realization of EoLC along the residents' and their families' wish as well as securing of quality [1]. The EoLC at nursing home depends on cooperation among various professions such as nurses, care workers, consultants, dieticians and so on as well as physicians. Therefore, it is necessary to explore measures so that all the staff members including specialists at nursing home can promote this cycle with themselves at each position assuming their roles. The authors believe that it is desirable to deepen mutual understanding among the professions, unify their policies, and establish a collaboration system of EoLC utilizing the advantages of the facilities. For the EoLC at nursing homes that has been performed based on trial and error since the setting of additional aid for EoLC of 2006. It is time to focus on evaluating quality of EoLC from different 
angles comprehensively in step with the establishment of environment to improve quality of care practice and care effects, system establishment, in other words. Therefore, as security of regular nurses was included in the requirement for acquiring additional aid for EoLC so as to secure the quality of EoLC, responsibility for the PDCA cycle propulsion assumed by nursing professions engaged in medical service at nursing home, which is place for living of elderly people, is large. However, a survey conducted by Japanese Council of Senior Citizens Welfare Service [2] revealed that most nursing homes were suffering from short of nursing care staff, and their nursing staff systems are insufficient. Furthermore, the cooperation between medical service and nursing was maintained based on on-call systems operated only by one nursing staff member in approximately $25 \%$ of the facilities, indicating that burdens on nursing staff are extremely heavy in the present situation. Moreover, according to Ueda, et al. [3], in a nursing home in a certain prefecture, approximately $60 \%$ of nurses provide support for substituted judgment for families of the residents who have difficulty in declaring their intentions while they are not confident on if the decisions are best ones that the residents wish. The reasons for the above are that most facilities do not confirm contents of periodical instructions described in preliminary instruction $s$ and families and staff members cannot confirm ideal EoLC that the elderly people wish continuously during the period from their entrance to the terminal phase. It has been reported that while elderly residents cannot communicate because of dementia and consciousness disturbance at a terminal phase in most cases, nurses feel difficulty in assuming the role of providing support for substitute's judgment for their families. The role and responsibility of the nursing staff members engaged in medical service for EoLC in facilities are important for quality of EoLC even though they work with few members, and it is necessary to be involved with evaluation and improvement from an administrative viewpoint in construction of an EoLC system. Ministry of Health, Labour and Welfare proposed management service and leadership expected for nurses in nursing homes, appropriate response and physical assessment of elderly people, and education contents about network construction and personnel development, as contents that need to be included in nursing leader trainings for future nursing homes [4]. These training contents back up the fact that nurses are required not only to secure care quality in given environment but also to have an ability to lead peaceful EoLC that residents expect in a nursing home cooperating with other professions smoothly. However, there have been only few studies on evaluation and improvement of EoLC systems by nursing managers in nursing homes. Therefore, the main purpose of this study is to verify statistically, based on the Donabedian theory to evaluate medical quality, practice situation of the PDCA cycle of EoLC nursing care, nursing competence of a nursing team and relationship of EoLC achievement as a quality estimation model of EoLC from the viewpoint of the nursing manager in a nursing home. Moreover, this study aimed at, as a second purpose, clarifying factors for improving nursing competence based on the verification results of the model. The authors believe the results of this study provides nursing managers with suggestions about policy for visualizing the role of nursing to promote EoLC in a nursing home and improving the team power in EoLC in such facilities while they contributes to securing quality of EoLC of elderly people spending their final moment in facilities, which are place for living.

\section{Theoretical Framework of This Study}

Figure 1 shows the conceptual framework of this study. Avedis Donabedian proposed to evaluate quality of health care services by three aspects of "structure" "process" and "result" [5]. This is a theory to evaluate quality based on the functional relationship that "structure" that shows human material resources or systems required for medical care system influences "process" indicating medical technology and care technique including personal relationships and ethics while it also influences positively "result" that indicates health change brought to patients by medical care. In other words, it is a frame that statistically visualizes quality evaluation using the causal model of "structure" -> "process" -> "result". In our precedent study [6], the authors, based on the Donabedian theory, verified the causal model to evaluate quality of EoLC from the viewpoint of nursing, which is based on the idea that an environmental structure of EoLC in a nursing home influences nursing competence and furthermore achievements. The environmental structures above consisted of the number of nurses in the nursing home, presence of unit care support, frequency of EoLC training, frequency of conference after EoLC, presence of EoLC manual and presence of advance instruction book. These environmental structures were environmental factors to improve nursing competence for EoLC in a nursing home and were included in the items of the PDCA cycle of EoLC nursing care that Ministry of Health, Labour and Welfare [1] presented. Moreover, the PDCA cycle of EoLC nursing care has been proposed as a new measure that strengthens more the EoLC systems in nursing homes, aiming at construction of an environment that provides high quality EoLC by propulsion of its circulation. Therefore, in this study, the authors captured items of the PDCA cycle of EoLC nursing care as environmental structures of EoLC and verified its influence on nursing competence for EoLC statistically. Further, the PDCA cycle of EoLC nursing care presented by Ministry of Health, Labour and Welfare consists of [Maintenance of the system] as Plan indicating the facilities' EoLC policy, [EoLC nursing care] as Do indicating the support to the final moment that the elderly persons wish based on the plan related to EoLC nursing care, [Reflection] as 
Check indicating verification of EoLC nursing care through conference, or grasp of mental strain by staff members and their support, and [Improvement of the system] as Action indicating review of guideline and system of EoLC [7]. This study classifies environmental-structural factors using this frame. The nursing competence for EoLC scale applied to "Process" is as scale to measure nursing competence for EoLC in nursing homes, and reliability and validity of the scale has already been confirmed [8]. While self-evaluation of nursing professions was used in the precedent study, nursing competence of the entire nursing team in the facilities was assessed by nursing managers in this study. Achievement of EoLC including the number of times of EoLC practiced was applied to "Result". In other words, the authors presumed that under the assumption that a model that the items of the PDCA cycle influence nursing competence for EoLC as well as the achievement is verified, the entire model would act as an index for evaluating EoLC quality. For achievement of EoLC, which was assumed as "Result", objective real numbers such as the real number of times of EoLC realized along the residents' wish, the number of cases that families were able to participate in EoLC and accompany the death (good or bad of the family relations does not matter), and additional aid for EoLC calculated were introduced. After EoLC, evaluation for satisfaction level cannot be done by the residents who received care. Moreover, there is a lot of cases that satisfaction levels reported by families may not be true due to their hesitation to the staff members of the facilities who provided support till the death in the case of EoLC for senility of an elderly person who finished their long life. Therefore, we verified the "result" obtained in this study by achievement (real number) not by subjective assessment based on satisfaction levels

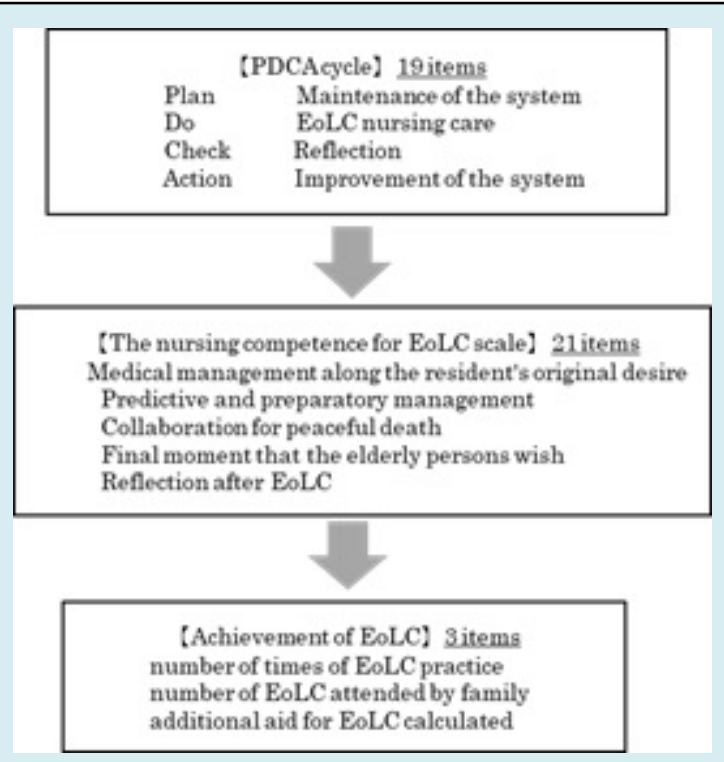

Figure 1: The conceptual framework of this study.

\section{Method}

\section{Subjects}

Additional aid for EoLC was calculated for 4390 nursing home facilities registered at Japanese Council of Senior Citizens Welfare Service, 1000 nursing home facilities with more than five nurses dealing with more than 10 case of EoLC practice per year were systematically sampled, and nursing managers at the facilities were employed as subjects. The number five for number of nurses at the facilities is the average value of the number of nursing professions in the precedent study [8].

\section{Investigation Method}

Data collection method: Study request documents and questionnaires were sent to the facility managers and nursing managers of the selected 1000 nursing homes, together with return envelope, as a self-administered anonymous questionnaire survey. Senders and respondents were all anonymous, and it was regarded that study consent was obtained from the sender at the time when their questionnaire was returned. In the process of data collection, the researchers prepared sampling data of the facilities, study request document and questionnaire, and data shipment and collection, and data entry were done by a special company.

Survey content: The number of survey items of the questionnaire was 49 in total. The target period for real numbers was 2014.

\section{$>$ Nine items for attributes of the facilities outline (including achievement) and subject}

The items about the facilities outline were (1) number of residents, (2) number of nursing professionals, (3) number of caring professionals, (4) number of discharge by death, (5) number of times of EoLC practice, (6) number of EoLC attended by family and (7) additional aid for EoLC calculated. The items about subjects' attributes were two items of age and years of experience in EoLC in the facilities

\section{$>$ Nineteen question items about the PDCA cycle of EoLC nursing care}

In this study, 19 question items to measure presence, frequency and degree for the items of PDCA cycle were made as follows.

\section{- Maintenance of the system: Plan}

(1) presence of guideline for EoLC, (2) sufficiency of description of guideline to residents and their family, (3) frequency of staff member training for EoLC, (4) presence of 24-hour communication system for physicians and nursing staff members, (5) sufficiency of the system in which caring 
staff members can handle various situations even when in nursing members are absent, (6) presence of private rooms or resting rooms used for EoLC and (7) presence of cooperation with hospitals for emergency transportation.

\section{- EoLC: Do}

(8) sufficiency of planning for EoLC nursing care of each user, (9) sufficiency of explanation of plans to residents and their family, (10) presence of information on daily change in residents' symptoms to be shared among different professions, (11) presence of documents for information supplements about EoLC for residents and their family (e.g. leaflets) and (12) nursing staff system for night and (13) sufficiency of support for grief of residents' family.

\section{- Reflection: Check}

(14) Frequency of care conference after EoLC, (15) degrees of grasping mental strain of the staff members involved in EoLC and (16) sufficiency of support for mental strain of the staff members involved in EoLC.

\section{- Improvement of the system: Action}

(17) progress of review of guideline for EoLC, (18) frequency of briefing session about EoLC and (19) presence of educational activities of EoLC in the local community including residents and their family.

The items that asked presence were scored by two-point scale of Yes $=2$ points and No $=1$ point, and those that asked frequency and sufficiency were scored by three-point scale of No $=1$ point, insufficient $=2$ points and sufficient $=3$ points.

- Scale to measure nursing competence for EoLC in nursing home: 5 factors and 21 items

This scale is to measure nursing competence to practice peaceful EoLC for residents in a nursing home. Its reliability and validity have already been confirmed [8].

This scale consists of 5 factors and 21 items, based on four-point scale of "Not done = 1 point " $t$ - "Sufficiently done $=4$ points".

\section{Analysis Method}

- Descriptive statistics were calculated for each item.

- Pearson's correlation coefficients were calculated for items of the PDCA cycle, factors for nursing competence for EoLC in nursing home and achievement of EoLC.

- Confidence coefficient of nursing competence for EoLC in nursing home (Cronbach's $\boldsymbol{\alpha}$ coefficient: hereinafter called $\boldsymbol{\alpha}$ coefficient) was calculated.

- The hypothesis of this study was multi-indicator model, that shows relationship between causal sequence of the constructive concepts of "PDCA cycle", "Nursing competence for EoLC" and "Achievement of EoLC" and observation variables of each constructive concept in one path diagram.

Therefore, reliability and validity of 19 items of the PDCA cycle and the scale to measure nursing competence for EoLC in this study were confirmed by $\alpha$ coefficients and a confirmatory factor analysis, and a covariance structure analysis was conducted for the hypothesis model after that. In the covariance structure analysis, relationship between constructive concepts, which cannot be directly observed as numerical value $s$, and observation variable, which are measurable as numerical values is expressed with a path diagram. Moreover, relationship between constructive concepts can be described [9]. $\chi 2 /$ df value, GFI, AGFI, IFI, CFI were used as fit indices. The statistical analysis software SPSS ver19.0 for Windows, Amos ver. 20 was used for these data analysis.

\section{Ethical Consideration}

This study was approved by Ethical Review Board of Seirei Christopher University. In this study, in order to request their nursing managers of nursing homes for cooperation with this study, documents of study description and request for cooperation with study cooperation were sent to the facility managers, so as to obtain understanding of the nursing manager on cooperation with the study. Moreover, the document clearly described that questionnaire was anonymous with no respondents' signature and senders' names required, the study participation was based on free will and nonparticipation would not cause any disadvantage, anonymity was observed for any cases including publication of results, the data were to be strictly stored and discarded at the time of completion of the study. Furthermore, it was regarded that study consent was obtained from the sender at the time when their questionnaire was returned.

\section{Results}

\section{Outline of Facilities and Subjects' Attributes (Tables 1 and 2)}

As a result of mail questionnaire survey, answer sheets were collected from 267 subjects with the collection ratio of $26.7 \%$. Effective answers were obtained from 256 subjects, with the response rate of $95.8 \%$. Approximately $50 \%$ of the nursing managers, the subjects for this study, were in their 50 s, and $30 \%$ of them were in their 40 s. Years of experience in EoLC at facilities were equally approximately 30\% for each of less than 5 years, 5-9 years and 10 years or more. The number of residents at the facilities varied from 25 to 300, the average of 78.7+-31.3. The average number of nursing professionals was 5.8+-2.1. Moreover, the average number of discharges by death was $14.2+-9.6$, that of EoLC practice was 
8.5+-7.5, that of EoLC attended by family was $4.4+-5.2$ was,

and that of additional aid for EoLC calculated was 5.3+-6.4.

\begin{tabular}{|c|c|c|}
\hline \multicolumn{2}{|c|}{ Number of People } & $\%$ \\
\hline \multicolumn{2}{|c|}{ age } \\
\hline $30 \mathrm{~s}$ & 30 & 11.7 \\
\hline $40 \mathrm{~s}$ & 71 & 27.7 \\
\hline $50 \mathrm{~s}$ & 122 & 47.7 \\
\hline $60 \mathrm{~s}$ & 32 & 12.5 \\
\hline other & 1 & 4 \\
\hline \multicolumn{1}{|c|}{ years of experience in EoLC } \\
\hline less than 5 years & 93 & 36.3 \\
\hline 10 years or more & 76 & 29.7 \\
\hline
\end{tabular}

Table 1: Subjects attributes.

\begin{tabular}{|c|c|c|}
\hline & Average & SD \\
\hline Number of residents & 78.7 & 31.3 \\
\hline Number of nursing professionals & 5.8 & 2.1 \\
\hline Number of caring professionals & 41.1 & 18.5 \\
\hline Number of discharge by death & 14.2 & 9.6 \\
\hline Number of times of EoLC pratice & 8.5 & 7.5 \\
\hline Number of EoLC attended by family & 4.4 & 5.2 \\
\hline Additional aid for EoLC calculated & 5.3 & 6.4 \\
\hline
\end{tabular}

Table 2: Outline of Facilities.

\section{Practice Situation of Eolc Nursing Care PDCA Cycle (Table 3)}

In an evaluation of the practice situation of the items in the PDCA cycle by the nursing manager of the special elderly nursing home, 91\% answered "Yes (they did)" for presence of EoLC guideline.

\begin{tabular}{|c|c|c|}
\hline The items of PDCA cycle & \multicolumn{1}{|c|}{ Number of people } & \% \\
\hline [Maintenance of the system: Plan] & $\boldsymbol{\alpha}=.521$ & 8.6 \\
\hline No & 22 & 91 \\
\hline Yes & 233 & 0.4 \\
\hline No answer & 1 & 9.8 \\
\hline No & 25 & 35.2 \\
\hline not enough & 90 & 52.7 \\
\hline sufficient & 135 & 2.3 \\
\hline No answer & 6 & 15.6 \\
\hline 3. Frequency of staff member traning for EoLC & 40 & \\
\hline
\end{tabular}




\begin{tabular}{|c|c|c|}
\hline Once a year & 152 & 59.4 \\
\hline Once every six month & 54 & 21.1 \\
\hline Monthly & 3 & 1.2 \\
\hline No answer & 7 & 2.7 \\
\hline \multicolumn{3}{|c|}{ 4. Presence of 24-hour communication system for physicians and nursing staff members } \\
\hline No & 8 & 3.1 \\
\hline Yes & 248 & 96.9 \\
\hline \multicolumn{3}{|c|}{$\begin{array}{l}\text { 5. Sufficiency of the system in which caring staff members can handle various situations even when in nursing mem } \\
\text { bers are absent }\end{array}$} \\
\hline No & 22 & 8.6 \\
\hline not enough & 129 & 50.4 \\
\hline Sufficient & 100 & 39.1 \\
\hline No answer & 5 & 2 \\
\hline \multicolumn{3}{|l|}{ Presence } \\
\hline \multicolumn{3}{|c|}{ 6. Presence of private rooms or resting rooms used for EoLC } \\
\hline No & 20 & 7.8 \\
\hline Yes & 235 & 91.8 \\
\hline No answer & 1 & 0.4 \\
\hline \multicolumn{3}{|c|}{ 7. presence of cooperation with hospiotals for emergency transportation } \\
\hline No & 35 & 13.7 \\
\hline Yes & 219 & 85.5 \\
\hline No answer & 2 & 0.8 \\
\hline [EoLC nursing care: Do] & $\alpha=.623$ & \\
\hline \multicolumn{3}{|c|}{ 8. Sufficiency of planning for EoLC nursing care of each user } \\
\hline No & 23 & 9 \\
\hline not enough & 51 & 19.9 \\
\hline sufficient & 181 & 70.7 \\
\hline No answer & 1 & 0.4 \\
\hline \multicolumn{3}{|c|}{ 9. Sufficiency of explanation of plans to residents and their family } \\
\hline No & 18 & 7 \\
\hline not enough & 50 & 19.5 \\
\hline Draft & 186 & 72.7 \\
\hline No answer & 2 & 0.8 \\
\hline \multicolumn{3}{|c|}{ 10. Presence of information on daily change in residents' symptons to be shared among different professions } \\
\hline No & 10 & 3.9 \\
\hline Yes & 245 & 95.7 \\
\hline No answer & 1 & 0.4 \\
\hline The items of PDCA cycle & Number of people & $\%$ \\
\hline \multicolumn{3}{|c|}{ 11. Presence of documents for information supplements about EoLC for residents and their family (e.g. Leaflets) } \\
\hline No & 107 & 41.8 \\
\hline Yes & 143 & 55.9 \\
\hline No answer & 6 & 2.3 \\
\hline 12. Nursing staff system for night & & \\
\hline
\end{tabular}




\begin{tabular}{|c|c|c|}
\hline No night shift & 13 & 5.1 \\
\hline on-call & 22.8 & 89.1 \\
\hline Night duty & 8 & 3.1 \\
\hline Other & 7 & 2.7 \\
\hline \multicolumn{3}{|l|}{ 13. Sufficiency of support for grief of residents family } \\
\hline No & 26 & 10.2 \\
\hline Not enough & 152 & 59.4 \\
\hline Sufficient & 76 & 29.7 \\
\hline No answer & 2 & 0.8 \\
\hline [Reflection: Check] & $\alpha=.264$ & \\
\hline \multicolumn{3}{|l|}{14 frequency of care conference after EoLC } \\
\hline No & 63 & 24.6 \\
\hline Not enough & 52 & 20.3 \\
\hline Sufficient & 139 & 54.3 \\
\hline No answer & 2 & 0.8 \\
\hline \multicolumn{3}{|c|}{ 15. Degrees of grasping mental strain of the staff members involved in EoLC } \\
\hline No & 19 & 7.4 \\
\hline Instead & 145 & 56.6 \\
\hline Sufficient & 92 & 35.9 \\
\hline \multicolumn{3}{|c|}{ 16. Sufficiency of support for mental strain of the staff members involved in EoLC } \\
\hline No & 44 & 17.2 \\
\hline Not enough & 181 & 70.7 \\
\hline Sufficient & 31 & 12.1 \\
\hline [Improvement of the system: Action] & $\alpha=.447$ & \\
\hline \multicolumn{3}{|l|}{ 17. Progress of review of guidelines for EoLC } \\
\hline No & 74 & 28.9 \\
\hline Better to do & 85 & 33.2 \\
\hline Already doing & 93 & 36.3 \\
\hline No answer & 4 & 1.6 \\
\hline \multicolumn{3}{|l|}{ 18. Frequency of briefing session about EoLC } \\
\hline No & 118 & 46.1 \\
\hline Report on-site & 125 & 48.8 \\
\hline Report outside the facility & 12 & 4.7 \\
\hline No answer & 1 & 4 \\
\hline \multicolumn{3}{|c|}{ 19. Presence of educational activities of EoLC in the local community including residents and their family } \\
\hline No & 208 & 81.3 \\
\hline Yes & 48 & 18.8 \\
\hline
\end{tabular}

Table 3: Practice situation of EoLC nursing care PDCA cycle.

For description of guideline to residents and their family, $52.7 \%$, the largest portion, answered "Sufficient", 35.2\% answered "Slightly insufficient" and 9.8\%, the smallest portion, answered "No (description)". For frequency of staff member training for EoLC, 59.4\%, the largest portion, answered "Once a year" while 15.6\% answered "No". For presence of 24-hour communication system, 96.9\% answered "Yes", while for the system in which caring staff members can handle various situations even when in nursing members are absent, 50.4\%, the largest portion, 
answered "Slightly insufficient" and 8.6\% answered "No". For presence of private rooms or resting rooms used for EoLC, 91.8\% answered "Yes" and for presence of cooperation with hospitals for emergency transportation, 85.5\% answered "Yes". For sufficiency of planning for EoLC nursing care of each user, 70.7, the largest portion, answered they "Always plan". For sufficiency of explanation of plan to residents and their family, $72.7 \%$ answered they "Do sufficiently" while 7\% (18 subjects) answered they did not explain at all. For presence of information on daily change in residents' symptoms to be shared among different professions, 95.7\% answered "Yes". For presence of documents for information supplements about EoLC for residents and their family, 55.9\% answered "Yes" while $41.8 \%$ answered "No". For nursing staff system for nighttime, $89.1 \%$, the largest portion, answered they had "On-call systems". For sufficiency of support for grief of residents' family, 59.4\%, the largest portion, answered "Slightly insufficient". For frequency of care conference after EoLC, 54.3\% answered those they had conference "Every time" and 24.6\% answered "No". For degrees of grasping mental strain of the staff members involved in EoLC, $92.5 \%$ answered that they had mental strain "To some extent" or "Very much". For sufficiency of support for mental strain of the staff members involved in EoLC, 70.7\%, the largest portion, answered "Slightly insufficient". For progress of review of guideline for EoLC, 36.3\%, the largest portion, answered that they were "Reviewing". For frequency of briefing session about EoLC, 48.8\% answered that they "Reported in the facilities" while $46.1 \%$ answered that they "Did not report". For presence of educational activities of EoLC in the local community including residents and their family, $81.3 \%$, the largest portion, answered that "No".

\section{Evaluation of Nursing Competence for Eolc in Nursing Team (Table 4)}

Average values and standard deviations of the scores for nursing competence for EoLC in nursing teams evaluated by nursing managers are shown below. The average score for [Collaboration for peaceful death], which is a factor for nursing competence for EoLC was 3.07+-.55 points. [Final moment that the elderly persons wish] was 2.88+-.65 points, [Medical management along the resident's original desire] was $2.99+-.54$ points, [Predictive and preparatory management] was $3.37+-.50$ points, and [Reflection after EoLC] was $2.77+-.79$ points. The highest score was seen in [Predictive and preparatory management] followed by [Collaboration for peaceful death]. These two factors presented scores over 3 points (achievement level: fairly good). On the other hand, the lowest score was seen in [Reflection after EoLC], [Final moment that the elderly persons wish] and [Medical management along the resident's original desire]. In particular, standard deviation is relatively high for [Reflection after EoLC], all of the four subitems were below 3 points.

\begin{tabular}{|c|c|c|c|}
\hline & Average & SD & $\alpha$ \\
\hline [Collaboration for peaceful death] & 3.07 & 0.55 & .824 \\
\hline Observation and assessment of symptoms and pain characteristics to the end-of-life stage & 2.93 & 0.7 & \\
\hline Prediction of entry to the terminal stage & 3.18 & 0.65 & \\
\hline Close collaboration with care workers specializing in life support & 3.11 & .65 & \\
\hline $\begin{array}{l}\text { Standardization of end of-life care policies in consideration of the opinions of the other } \\
\text { professionals }\end{array}$ & 3.07 & 0.71 & \\
\hline [Final moment that the elderly persons wish] & 2.88 & 0.65 & 0.818 \\
\hline Consideration of requests to residents religious customs and beliefs & 2.85 & 0.83 & \\
\hline Reflection of resident interests, preferences, life history, etc., in end-of-life care plus & 2.86 & 0.76 & \\
\hline $\begin{array}{l}\text { Inference and application of resident wishes based on individual characteristics in cases } \\
\text { where direct communication is impractical }\end{array}$ & 2.93 & .67 & \\
\hline [Medical management along the resident's original desire] & 2.55 & 0.54 & 0.785 \\
\hline Provision of care prioritizing resident wishers as much as possible & 3.26 & 0.69 & \\
\hline $\begin{array}{l}\text { Development relationships supporting free expression among residents prior to the } \\
\text { terminal stage }\end{array}$ & 2.87 & .66 & \\
\hline $\begin{array}{l}\text { Provision of opportunities for residents and family to express wishes regarding end-of-life } \\
\text { care }\end{array}$ & 2.88 & .73 & \\
\hline Administration of pain control in collaboration with a doctor & 3.04 & .80 & \\
\hline Review of unnecessary treatment and testing once the terminal stage has been reached & 3.09 & .77 & \\
\hline
\end{tabular}




\begin{tabular}{|c|c|c|c|}
\hline [Predictive and preparative management] & 3.37 & 0.5 & 0.885 \\
\hline Prediction of sudden night time changes based on daytime wellbeing among residents & 3.17 & .60 & \\
\hline Prediction of potential symptoms and advance medical consultation & 3.16 & 0.63 & \\
\hline $\begin{array}{l}\text { Provision of information to care workers on the next night shift regarding the possibility of } \\
\text { sudden changes in resident conditions }\end{array}$ & 3.40 & 0.59 & \\
\hline Provision of information to residents and family on medical services available at the facility & 3.54 & 0.6 & \\
\hline Checking of resident/family wishers regarding life-prolonging procedures & 3.55 & 0.6 & \\
\hline [Reflection after EoLC] & 2.77 & 0.79 & .957 \\
\hline Recall and assessment of whether resident passing was painless and natural & 2.84 & 0.86 & \\
\hline $\begin{array}{l}\text { Recall and assessment regarding the effects of care administered and the status of the } \\
\text { resident's body }\end{array}$ & 2.76 & 0.84 & \\
\hline $\begin{array}{l}\text { Recall of exchanges with family and their relations, and assessment on the appropriateness } \\
\text { of support provided }\end{array}$ & 2.73 & .81 & \\
\hline Recall and assessment on whether end-of-life care in line with resident wishes was provided & 2.75 & .84 & \\
\hline
\end{tabular}

Table 4: Evaluation of nursing competence for EoLC in nursing team $(\mathrm{N}=256)$.

\section{Relationship between PDCA Cycle-Related Items, and Nursing Competence for Eolc and Achievement (Table 5)}

Since the variables measured in this study almost presented normal distribution, Pearson' correlation coefficients were calculated. For the PDCA cycle-related items, the factors of nursing competence for EoLC for which significant correlation $(.4<\mathrm{r}<=.7)$ was recognized were [Collaboration for peaceful death], [Medical management along the resident's original desire] and [Reflection after EoLC] for (2) sufficiency of description of guideline to residents and their family. For (8) sufficiency of planning for EoLC nursing care of each user, the factor was [Reflection after EoLC], and for (9) sufficiency of explanation of plans to residents and their family, the factors were [Final moment that the elderly persons wish], [Medical management along the resident's original desire], [Predictive and preparatory management] and [Reflection after EoLC]. [Collaboration for peaceful death], [Final moment that the elderly persons wish] and [Medical management along the resident's original desire] were obtained as factors for 13 sufficiency of support for grief of residents' family, and [Final moment that the elderly persons wish] and [Reflection after EoLC] were obtained as factors for (14) frequency of care conference after EoLC. The correlation between the [Reflection after EoLC] and frequency of care conference after EoLC was strongest. For (16) sufficiency of support for mental strain of the staff members involved in EoLC, [Collaboration for peaceful death], [Final moment that the elderly persons wish] and [Medical management along the resident's original desire] were obtained as factors. On the other hand, achievement of EoLC showed significant but weak correlation with several PDCA cycle-related items $(.2<\mathrm{r}<=.4)$. The number of EoLC attended by family and additional aid for EoLC calculated presented weak correlation with (2) sufficiency of description of guideline to residents and their family, while all items showed weak correlations with (8) sufficiency of planning for EoLC nursing care of each user, (9) sufficiency of explanation of plan to residents and their family and (19) presence of educational activities of EoLC in the local community including residents and their family. Moreover, (17) progress of review of guideline for EoLC and 18 frequency of briefing session about EoLC presented weak correlations with the number of EoLC practice and that of EoLC attended by family, and additional aid for EoLC calculated, respectively.

\begin{tabular}{|c|c|c|c|c|c|c|c|c|c|c|c|c|c|c|c|c|c|c|c|}
\hline & \multicolumn{8}{|c|}{ [Maintenance of the system: Plan] } & \multicolumn{5}{|c|}{ [EoLC nursing Care: Do] } & \multicolumn{3}{|c|}{$\begin{array}{l}\text { [Refrection: } \\
\text { Check] }\end{array}$} & \multicolumn{3}{|c|}{$\begin{array}{l}\text { [Improvement of the } \\
\text { system: Action] }\end{array}$} \\
\hline & 1 & 2 & 3 & 4 & 5 & 6 & 7 & 8 & 9 & 10 & 11 & 12 & 13 & 14 & 15 & 16 & 17 & 18 & 19 \\
\hline \multicolumn{20}{|c|}{ Attribute } \\
\hline age & 0.043 & -0.05 & 0.014 & -0.027 & 0.018 & 0.026 & 0.098 & 0.076 & 0.057 & 0.049 & 0.066 & -0.079 & 0.061 & $186^{* *}$ & 0.066 & 0.017 & 0.081 & $.193^{* *}$ & 0.095 \\
\hline Years of experience in EoLC & 0.114 & $.174^{*}$ & 0.072 & 0.102 & 0.098 & 0.024 & 0.038 & 0.117 & $.150 *$ & 0.035 & 0.002 & 0.045 & 0.067 & 0.023 & 0.096 & 0.084 & $.150^{*}$ & $.170^{* *}$ & $.169^{*}$ \\
\hline
\end{tabular}




\begin{tabular}{|c|c|c|c|c|c|c|c|c|c|c|c|c|c|c|c|c|c|c|c|}
\hline \multicolumn{20}{|c|}{ Online of facilities } \\
\hline number of residents & 0.032 & -0.062 & 0.014 & 0.048 & 0.106 & 0.051 & -0.172 & 0.042 & 0.248 & 0.002 & -0.035 & 0.099 & 0.006 & 0.083 & -0.021 & 0.014 & 0.052 & 0.026 & 0.03 \\
\hline $\begin{array}{c}\text { number of running } \\
\text { professionals }\end{array}$ & 0.021 & 0.001 & -0.25 & 0.058 & $218^{* *}$ & -0.38 & 0.008 & 0.012 & -0.021 & -0.01 & -0.038 & $221^{* *}$ & -0.024 & 0.041 & -0.121 & 0.08 & 0.046 & 0.114 & 0.023 \\
\hline $\begin{array}{c}\text { number of caring } \\
\text { professionals }\end{array}$ & 0.045 & -0.039 & 0.036 & 0.042 & 0.075 & -0.006 & -0.111 & 0.082 & 0.02 & -0.038 & -0.01 & -115 & 0.033 & 0.182 & 0.007 & 0.039 & 0.115 & 0.063 & 0.022 \\
\hline $\begin{array}{c}\text { number of discharge by } \\
\text { death }\end{array}$ & $.197^{* *}$ & $.124^{*}$ & 0.087 & $.153^{*}$ & 0.045 & $.140^{*}$ & -0.082 & $190 * *$ & $.202 * *$ & -0.003 & 0.018 & $.132^{*}$ & 0.041 & 0.117 & -0.05 & -0.012 & $.190^{* *}$ & 0.01 & $.145^{*}$ \\
\hline \multicolumn{20}{|c|}{ Achieve ement of EoLC } \\
\hline $\begin{array}{c}\text { number of times of EoLC } \\
\text { practice }\end{array}$ & $.185^{* *}$ & $.152^{*}$ & 0.095 & 0.122 & -0.001 & 0.153 & -0.078 & $264^{* *}$ & . $258^{* *}$ & 0.065 & 0.099 & $.148^{*}$ & 0.111 & $177^{* *}$ & -0.033 & 0.03 & $.226^{* *}$ & $.124^{*}$ & $.205^{* *}$ \\
\hline $\begin{array}{l}\text { number of EoLC attended } \\
\text { by family }\end{array}$ & 0.115 & $0.212^{* *}$ & $.171^{* *}$ & 0.079 & 0.067 & 0.122 & -0.022 & $251^{* *}$ & . $266^{* *}$ & 0.108 & 0.089 & 0.09 & $.151^{*}$ & $.232^{* *}$ & 0.032 & $167^{* *}$ & $.305^{* *}$ & $.175^{* *}$ & $.246^{* * *}$ \\
\hline $\begin{array}{l}\text { additional aid for EoLC } \\
\text { calculated }\end{array}$ & $.190^{* *}$ & $.229^{* *}$ & $.146^{*}$ & 0.127 & $.127 *$ & $.169^{* *}$ & 0.014 & $.356^{* *}$ & *.277** & 0.063 & $.152^{*}$ & 0.035 & $.155^{*}$ & $.283^{* *}$ & -0.112 & 0.101 & $.180^{* *}$ & $.233^{* *}$ & $.227^{* * *}$ \\
\hline \multicolumn{20}{|c|}{ Nursing components for EoLC in nursing team } \\
\hline $\begin{array}{c}\text { [collaboration for peaceful } \\
\text { death] }\end{array}$ & $.304^{* *}$ & $.423^{* *}$ & $.230^{* *}$ & $.290^{* *}$ & $.362^{* *}$ & 0.045 & 0.109 & $.378^{* *}$ & *.393** & $.307^{* *}$ & 0.116 & 0.063 & $.446^{* *}$ & $.340^{* *}$ & 0.036 & $.439 * *$ & $.280^{* *}$ & $.292^{* *}$ & $.201^{* *}$ \\
\hline $\begin{array}{l}\text { [Final moment that the } \\
\text { elderly persons wish] }\end{array}$ & $.272^{* *}$ & $.397^{* *}$ & $.150^{*}$ & $.209^{* *}$ & $.302^{* *}$ & 0.061 & 0.025 & $.370^{* *}$ & . $461^{* *}$ & $259 * *$ & $.169^{* *}$ & 0.075 & $.432^{* *}$ & $.406^{* *}$ & 0.11 & $.440^{* *}$ & $.309^{* *}$ & $.289^{* *}$ & $.177^{* *}$ \\
\hline $\begin{array}{l}\text { [Medical management } \\
\text { along the resident's original } \\
\text { desire }]\end{array}$ & $.346^{* *}$ & $.483^{* *}$ & $.188^{* *}$ & $230^{* *}$ & $.335^{* *}$ & 0.082 & 0.109 & $.389 * *$ & $.433^{* *}$ & $294^{* *}$ & $.229^{* *}$ & 0.069 & $.475^{* *}$ & $.327 * *$ & 0.004 & $449^{* *}$ & $.344^{* *}$ & $.218^{* *}$ & $.171^{* *}$ \\
\hline $\begin{array}{c}\text { [Predictive and preparatory } \\
\text { management] }\end{array}$ & $.262^{* *}$ & $.348^{* *}$ & $.139 *$ & $.240^{* *}$ & $.220^{* *}$ & $.134^{*}$ & 0.1 & $.311^{* *}$ & $.401 * *$ & $.335^{* *}$ & $.175^{* *}$ & 0.121 & $.365^{* *}$ & $.312^{* *}$ & 0.054 & $328^{* *}$ & $.241^{* *}$ & $.153^{*}$ & $.154^{*}$ \\
\hline \multirow[t]{2}{*}{ [Reflection after EoLC] } & $.261^{* *}$ & $.402^{* *}$ & $.284^{* *}$ & $.197^{* *}$ & $.224^{* *}$ & $.139 *$ & 0.099 & $.410^{* *}$ & $.441^{* *}$ & $287^{* *}$ & $.237^{* *}$ & 0.038 & $.355^{* *}$ & $.607^{* *}$ & 0.105 & $397^{* *}$ & $.324^{* *}$ & $.346^{* *}$ & $.185^{* *}$ \\
\hline & & & & & & & & & & & & & & & & & $\mathrm{P}<.05^{*}$ & $\mathrm{p}<.01^{* *}$ & $\mathrm{P}<.001 * * *$ \\
\hline
\end{tabular}

Table 5: Relationship between PDCAcycle-related items, and nursing competence for EoLC and achievement (Pearson's correlation coefficient).

\section{Reliability and Validity of Nursing Competence for Eolc Scale and PDCA Cycle Items (Tables 3 and 4) (Figures 2 and 3)}

Alpha coefficients were calculated for the reliability of the PDCA cycle in this study. [Maintenance of the system: as Plan] $\alpha=.521$, [EoLC: Do] $\alpha=.623$, [Reflection: Check] $\alpha$ $=.264$, and [Improvement of the system: Action] $\alpha=.447$. For adaptation of confirmatory factor analysis, $\chi 2 / \mathrm{df}=$ 2.072, GIF $=.885, \mathrm{AGIF}=.85, \mathrm{IFI} .833$ and $\mathrm{CFI}=.828$, and the coefficient of determination was $-.02<=\mathrm{R} 2>=.75$. The confidence coefficients of nursing competence for EoLC scale in this study were [Collaboration for peaceful death] $\alpha=.824$, [Final moment that the elderly persons wish] $\alpha=$
.818, [Medical management along the resident's original desire] $\alpha=.785$, [Predictive and preparatory management] $\alpha=.885$, and [Reflection after EoLC] $\alpha=.957$. For adaptation of confirmatory factor analysis, $\chi 2 / \mathrm{df}=2.625$, IGIF $=.835$, $\mathrm{AGIF}=.788$, IFI $=.924$ and CFI $=.923$, and the coefficient of determination was $.35<=\mathrm{R} 2<=.86$.

A: 【Collaboration for peaceful death】

B: 【Final moment that the elderly persons wish】

C: 【Medical management along the resident's original desire】

D:【Predictive and preparatory management】

E:【Reflection after EoLC】 


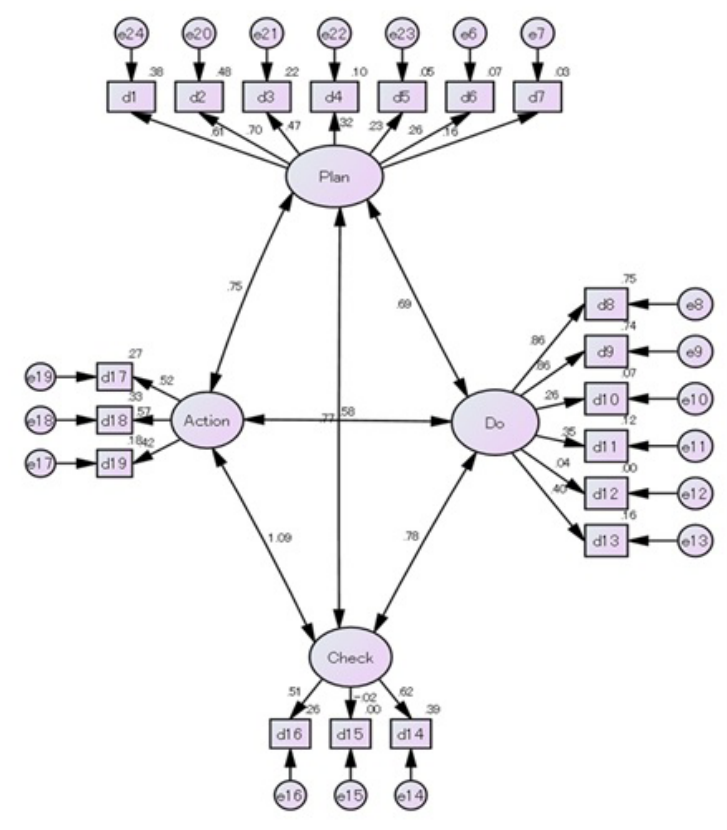

\begin{tabular}{|c|r|}
\hline CMIN/DF & 2.072 \\
\hline GFI & 0.885 \\
\hline AGFI & 0.85 \\
\hline IFI & 0.833 \\
\hline CFI & 0.828 \\
\hline
\end{tabular}

Figures 2: Results of confirmatory factor analysis of various items in the PDCA cycle.

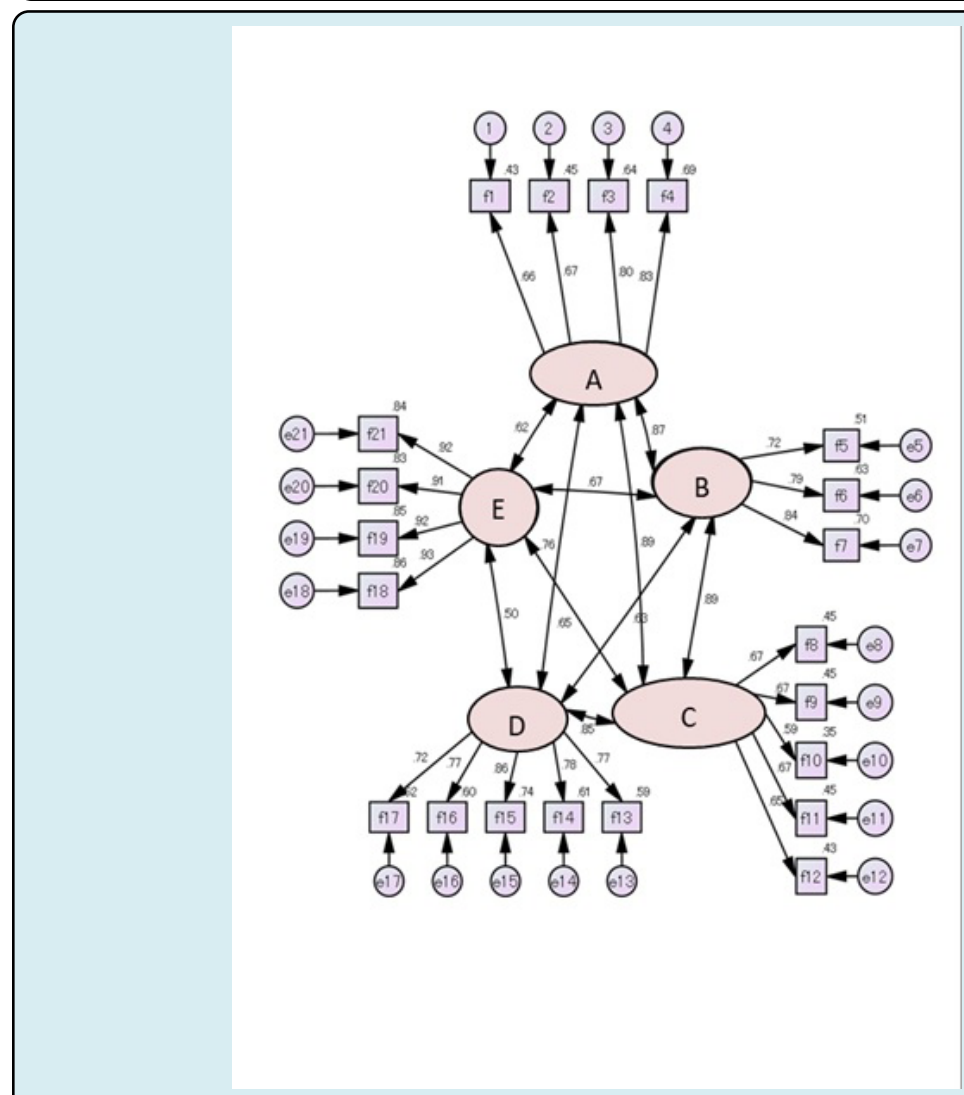

\begin{tabular}{|c|l|}
\hline CMIN/DF & 2.625 \\
\hline GFI & 0.835 \\
\hline AGFI & 0.788 \\
\hline IFI & 0.924 \\
\hline CFI & 0.923 \\
\hline
\end{tabular}

Figures 3: Results of confirmatory factor analysis of the nursing nursing ability scale of nursing. 


\section{Influence of Practice Situation of the PDCA Cycle on Nursing Competence for Eolc and Achievement (Figures 4)}

The authors, from the viewpoint of a nursing manager in a nursing home, repeatedly searched the model that showed relationships among practice situation of PDCA cycle, nursing competence of a nursing team and achievement of EoLC (the number of EoLC requested in facilities, the number of attendance by families and additional aid for EoLC calculated) while exploring its satisfactory adaptation that reached convergence. In the first search, the model was analyzed for all items though it did not converge. After that, several combinations were analyzed on the basis of exclusion of items with low correlation coefficients, in reference to PDCA cycle-related items, nursing competence for EoLC and achievement shown in in Table 5. As a result, we adopted the model that converged with the adaptation with $\chi 2 / \mathrm{df}=2.782$, GIF $=.826$, AGIF $=.788, \mathrm{IFI}=.833$ and CFI $=.831$ for which five items of (6) presence of private rooms or resting rooms used for EoLC, (7) presence of cooperation with hospitals for emergency transportation, (11) presence of documents for information supplements about EoLC for residents and their family (e.g. leaflets), (12) nursing staff system for night and (15) degrees of grasping mental strain of the staff members involved in EoLC were excluded. For these excluded items, the standardized estimate (hereinafter called path coefficient) of the confirmatory factor analysis was in the range of $-.02-.35$, and the coefficient of determination was also as low as $.00<=\mathrm{R} 2<=.12$. The path coefficient from PDCA to nursing competence as a latent variable was .79, and the coefficient of determination was $\mathrm{R} 2=.62$. Moreover, the paths coefficient from nursing competence to achievement of EoLC was .34 and the coefficient of determination was $\mathrm{R} 2=.11$. Path coefficients for subitems of the PDCA cycle were in the range of $.27-.70$. The items of over .5 that showed relatively high influence on nursing competence were (1) presence of guideline for EoLC, (2) sufficiency of description of guideline to residents and their family, (8) sufficiency of planning for EoLC nursing care of each user, (9) sufficiency of explanation of plans to residents and their family, (13) sufficiency of support for grief of residents' family, (14) frequency of care conference after EoLC and (16) sufficiency of support for mental strain of the staff members involved in EoLC. These coefficients of determinations are $.25<=\mathrm{R} 2<=$ .50 , which were higher than those of other items.
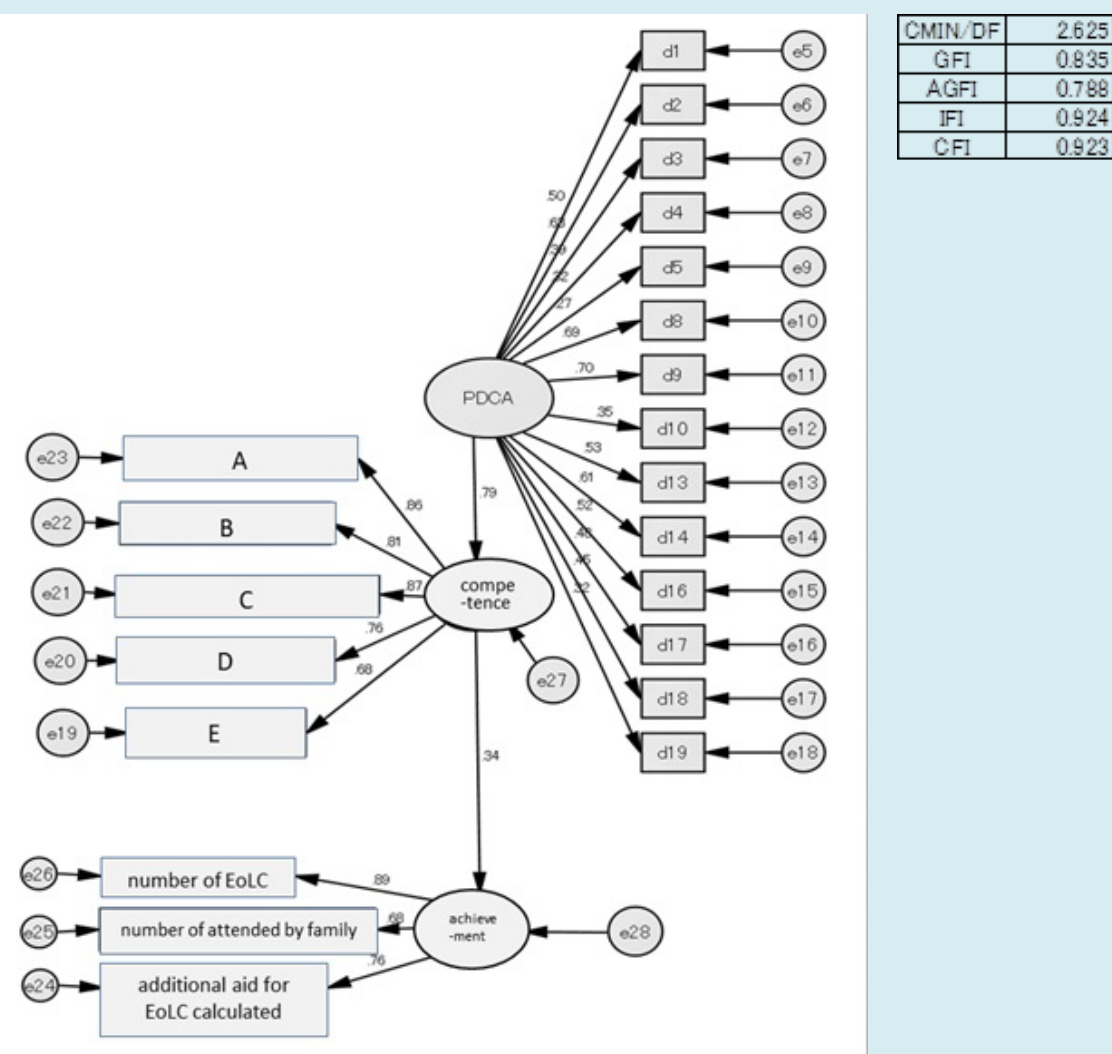

Figures 4: Covariance structure analysis of a hypothetical model. 


\section{Discussion}

\section{Verification of the Quality Estimation Model of the Eolc}

The subjects' attributes, evaluation of the items for the PDCA cycle of EoLC and achievement in this study were almost same level as the results in the survey on promotion of EoLC at nursing homes by Japanese Council of Senior Citizens Welfare Service (2015a) [2], indicating that there was no particular bias. Moreover, reliability of the nursing competence for EoLC scale in this study was .785<= $\alpha<=$ .957, which was sufficient, while that of the PDCA cyclerelated items was $.264<=\alpha<=.623$, indicating the reliability was not sufficient as a scale. The confirmatory factor analysis of validity revealed that adaptation of both scales converged with almost same values, proving satisfactory validity. On the other hand, binary variables were included in the 19 items of the PDCA cycle; we judged that the items were applicable to the model by setting the 19 items of the PDCA cycle as independent variables in this study. As for the result of the covariance structure analysis on the hypothesized EoLC quality evaluation model, verification of the relationships among the practice situation of the PDCA cycle of EoLC nursing care at the nursing homes, nursing competence of the nursing teams and achievement of EoLC has suggested that the results of this study are applicable to the EoLC quality evaluation model for nursing homes. Toyota (2013) [10] stated about the configuration of the causal model based on the structural equations that it is necessary to keep in mind that "The model is supported by real experience", "Description based on excellent theory that is understandable to most people has to be given", "The setting of causes in the theory configuration is done based on arbitrariness, and therefore the model has to be selected by the incentive that which aspects of the real world you want to understand phenomena from", and "The model needs to be based on the realistic request that what the causal model is constituted for". The causal model created in this study covers all the above points and therefore the model configuration is valid for evaluating quality of EoLC at a nursing home, we presume. However, the adaptation index of this model was not satisfactory. Its reasons, from a scientific viewpoint, presumably are that some of the PDCA cycle-related items have extremely low relationships with actual nursing service and that it was influenced by insufficiency of actions for the PDCA cycle in the facilities. The base for the former reason is that five items of (6) presence of private rooms or resting rooms used for EoLC, 7 presence of cooperation with hospitals for emergency transportation, (11) presence of documents for information supplements about EoLC for residents and their family (e.g. leaflets), (12) nursing staff system for night and (15) degrees of grasping mental strain of the staff members involved in EoLC, showed almost no correlations with factors of the nursing competence for EoLC in the correlation analysis with PDCA cycle-related items and 5 factors of the nursing competence (Table 5). The base for the latter reason is the items for which a number of nursing managers rated that actions were insufficient. Even for the items which were not excluded from the analysis, (13) sufficiency of support for grief of residents' family was approx. $80 \%$, (16) sufficiency of support for mental strain of the staff members involved in EoLC was approx. 90\%, and (19) presence of educational activities of EoLC in the local community including residents and their family was approx. $80 \%$, we infer. In other words, evaluation result that "the PDCA cycle of the EoLC nursing care in facilities is being promoted at the moment" is one of the factors that influenced the adaptation of the model. On the other hand, as an answer tendency of the five items excluded from the model verification, $91.8 \%$ had 6 private rooms or resting rooms used for EoLC and $85.5 \%$ had 7 cooperation with hospitals for emergency transportation. Moreover, $89.1 \%$ had on-call systems for (12) nursing staff system for night. For (15) degrees of grasping mental strain of the staff members involved in EoLC, $92.5 \%$ of the nursing managers answered "comparatively high" or "very high". For creation of (11) documents for information supplements about EoLC for residents and their family (e.g. leaflets), $55.9 \%$ answered that they had such documents presumably because this item was newly added the PDCA cycle in 2014 while this activity seemed to be still under promotion. These five items are the environmental conditions essential for securing EoLC quality in at nursing homes. Therefore, influence of these five items on nursing competence is to be focused on in the future study. On the other hand, the PDCA cycle of EoLC shows problems of the environment and system of EoLC practiced in nursing homes, which are imposed on specialists who support nursing homes such as counseling and nursing care staff members as well as facility managers. In particular, nursing care staff members who lack in knowledge and experience of EoLC actually practice EoLC with feeling of difficulty in medical procedures and feeling of difficulty in support at the time of emergency and sudden change (Nomura, 2017) [11]. The EoLC in nursing homes, which are place for living with no high-level medical facilities, is supported by care staff members working with the on-call system at night during which the nurses are absent in most facilities, and is realized by cooperation of nursing and caring staff members. Therefore, it is necessary to reflect the viewpoint of caring staff members who practice EoLC on the quality evaluation model, and develop indices for quality evaluation of EoLC that all staff members in nursing homes understand and utilize, while improving repeatedly the model who captures phenomena of EoLC at nursing homes from different angles. However, the model verification of this study has visualized the role of nursing to promote high quality EoLC in nursing homes. We have successfully elucidated the base for suggesting opinions for 
EoLC operation policy of the facilities from an administrative viewpoint and the position of nursing. From the above, each adaptation index did not present extremely bad values in the verification, and validity as an EoLC evaluation model, which allows scientific description of quality of EoLC environment and care at nursing homes, has been confirmed.

\section{Suggestion to Nursing Manager in PDCA Cycle of Eolc}

Here, the authors, based on the analysis, discuss characteristics of the factors of the PDCA cycle that is expected to exert a satisfactory influence on nursing competence for EoLC in a nursing home and suggestion to nursing managers for future problems in EoLC. One of the characteristics related to [Maintenance of the EoLC system], which is expected to exert a satisfactory influence on nursing competence, was that the facilities developed clear EoLC guidelines and explained sufficiently to the residents and their families about their principle. In the evaluation performed in this study, approximately $90 \%$ of the facilities developed EoLC guidelines while only a half of the nursing managers rated that the description to the residents and their families was sufficient. Nursing managers presumably have more opportunities to explain about EoLC for residents and their families than other nursing professions and it is inferred that they are uncertain if EoLC by the facilities are understood sufficiently by their description. According to the factual survey of Japanese Council of Senior Citizens Welfare Service (2015a) [2], approximately $70 \%$ of physicians, and counseling staff and nursing staff members assumed the role of explainer of EoLC guidelines almost equally and approximately $90 \%$ of them explained using the guideline developed in their facilities. Hashimoto and Ono (2014) [12] mentioned, as an obstructive factor of EoLC at a nursing home, the difficulty in relation with residents' families, who are to make decisions instead of the residents in EoLC period. The situation that families cannot understand natural death of an elderly person and that intentions cannot be unified among relatives is a specific factor seen comparatively often, they reported. In the description of the EoLC guideline, it is necessary to explain in detail how they reach death in the facilities, which are place for living, and care the elderly people and to what extent medical service can support them, as well as it is important to ascertain the timing to explain them during the process from admission to death. With that in mind, it is important to device, improve and seek easy-tounderstand description methods while always looking back on communication with the residents and their family. It is required for nursing managers of nursing homes to lead ACP (Advance care planning) in an attempt to cooperate with other professions and assume a role of adjusting for leading the team, so as to judge the need of support by physicians, counselors or nursing professions and provide
EoLC along the wish of residents and their family as much as possible while grasping information on them, we presume. As the second characteristic, factors related to [EoLC], which are expected to exert a satisfactory influence on nursing competence, included planning for EoLC, sufficient explanation of plans to residents and their family and support for grief of residents' family. In this investigation, approximately $70 \%$ of nursing managers rated "Sufficient" for both planning and explanation. The role that is important for nursing professions at nursing homes planning for care and its explanation is to obtain understanding on agreement for provision of care that would enable the elderly person to reach peaceful death without pain that the residents wish. Shimada et al. (2013) [13] states that communication with users and their families and improvement of care technique of members in the facilities would lead to a system that enables EoLC care. Quality of EoLC depends on if agreement and trust is obtained from the residents themselves and their families communication by specific planning of care and its explanation. It is required for nursing managers to assume a leadership role to let themselves and residents' families to think of ideal end of life in the residents' familiar environment so as to realize EoLC that the residents wish, we infer. Moreover, since "Support for grief of residents' family" for which approximately $70 \%$ of the nursing managers answered "Insufficient" showed correlations with [Collaboration for peaceful death], [Final moment that the elderly persons wish] and [Medical management along the resident's original desire], which are nursing competence, it is supposed that the process itself reduces grief of residents' family through performing careful planning while explaining it to the family and practicing EoLC with the families without regret. Fukuda et al. (2015) [14-17] stated that residents' families' participation in care and establishment of a system to accept cooperation are needed so as to enable residents' families to assume part of care whenever they wish, which will lead to satisfaction of EoLC of the residents and the families. On the basis of the above, it is important for nursing managers at nursing homes to recommend staff members to involve in residents' families to deepen the relationship with them even before EoLC. As the third characteristic, the factors related to [Reflection], which are expected to exert a satisfactory influence on nursing competence, were care conference after EoLC and support for mental strain of the staff members. In this study, approximately $70 \%$ of the facilities conducted care conferences after EoLC with each cycle or when needed while approximately $70 \%$ or more rated that the support for mental strain of the staff member was "Insufficient". While it is necessary to provide individual support for mental strain of staff members, the government recommends performing reflection of EoLC through conferences, which will lead to change in feelings and improvement of technique (Ministry of Health, Labour and Welfare, 2014) [1]. Regularization of care conference after EoLC is one of the goals while contents 
of the conference are more important. It is desired to forward effective conferences where the staff members express their feeling for residents and remorse of them including evaluation of the care, which leads to reduction of mental strain. Guidelines for how to lead effective care conferences specialized in EoLC at facilities are under development while it is necessary to regularize care conferences for EoLC of each staff member. Moreover, based on the idea that the conference itself will be evaluation of the EoLC, nursing managers are required to acquire management skill to work on quality evaluation and improvement of EoLC, we presume. Finally, [Maintenance of the system] such as EoLC training or 24-hour medical systems and [Improvement of the system] such as briefing sessions or enlightenment activities of EoLC to the local community as well as review of the guidelines were obtained as factors to exert an influence on nursing competence, though they did not influence as the above three characteristics did. The promotion of PDCA cycle for system construction and reinforcement of EoLC in facilities will be the base for high quality nursing practice in EoLC. Nursing managers are expected to work on construction and improvement of EoLC systems as a medical person of the facilities.

\section{Conclusions}

- Verification of relationship among achievement of EoLC, richness of the PDCA cycle of EoLC and nursing competence for EoLC has been suggested as a base a quality evaluation model of EoLC at nursing homes.

- The factors related to [Maintenance of the EoLC system], which are expected to exert a satisfactory influence on nursing competence for EoLC at nursing homes, was advocation of clear EoLC guidelines by facilities and sufficient explanation of the guidelines for a residents and their families.

- The factors related to [EoLC], which are expected to exert a satisfactory influence on nursing competence, included planning for EoLC, sufficient explanation of plans to residents and their family and support for grief of residents' family.

- The factors related to [Reflection], which are expected to exert a satisfactory influence on nursing competence for EoLC, were care conference after EoLC and support for mental strain of the staff members.

\section{Limitation of This Study and Future Problem}

The limitation of this study is that quality evaluation of EoLC nursing homes was performed by nursing managers. Quality of EoLC will be improved by searching for a method to return the results obtained in this study to clinics that is understood by different professions and promoting understanding and facilitation of cooperation among professions. Moreover, the collection ratio of this study was not high enough to be a national survey and therefore review of effective examination methods, and evaluation and review of quality of continuous EoLC will be future tasks. Furthermore, we are going to work on further studies so as to tune the results of this study into an EoLC quality evaluation model that applicable to facilities in Japan. In order to achieve it, as a subjective evaluation, which was not performed this time, it is necessary to verify and refine evaluation items as well as satisfaction levels of the entire staff members including residents families and care staff members for EoLC.

\section{Acknowledgement}

The authors sincerely thank the nursing managers of the nursing homes for their cooperation with the questionnaire survey of this time. Moreover, we sincerely thank teachers for their advices. This study was conducted with the financial aid of 2015 Japanese Council of Senior Citizens Welfare Service Research Project. This paper is the revised version of the contents presented at the 20th Annual Conference of The Japan Academy of Nursing Administration and Policies.

\section{References}

1. Ministry of Health, Labour and Welfare (2014) Reward and its standard of nursing home service. Social Security Council-The 112th nursing care benefit unit session document 1.

2. Japanese Council of Senior Citizens Welfare Service (2015a) Research study report on promotion of EoLC in nursing home and ideal medical cooperation. 2014 geriatric health project promotion grant project.

3. Ueda R, Ikino S (2016) Nursing profession's support to families of nursing home residents having difficulty in declaring their intentions for substituted judgment in the terminal phase. Japanese Society for the Study of Nursing and Social Work journal 21(2): 183-195.

4. Mitsubishi UFJ Research and Consulting (2016) Research study report on ideal high-quality care in nursing home. 2015 geriatric health promotion grants, Geriatric health promotion project.

5. Donabedian A (1990) Translated by Takahiro Azuma (2007) Definition and evaluation method of medical quality. Tokyo: iHope, Tokyo.

6. Omura M, Yamashita K, Nishikawa H (2015) Verification of the causal model to evaluate quality of EoLC in nursing homes from the viewpoint of nursing. Japan Society of Nursing Research journal 38(5): 13-22.

7. Japanese Council of Senior Citizens Welfare Service 
(2015b) EoLC care guideline/description support tool [2015 reward for nursing care for revised edition]. 2014 geriatric health project promotion grant project. Study on promotion of EoLC in nursing home and ideal medical cooperation.

8. Omura M, Yamashita K (2015) Development of nursing competence for EoLC Scale-Verification of reliability and validity. Japan Society of Nursing Research journal 39(1): 1-7.

9. Toyota H (2011) Covariance structure analysis-Structural equation modeling-Tokyo Tosho Co. Ltd, Tokyo.

10. Toyota $H$ (2013) Covariance structure analysis-Structural equation modeling-Asakura Publishing Co. Ltd, Tokyo.

11. Nomura 0 (2017) Trend and problem of the studies on recognition of care staff member for death and terminal care at nursing homes-Literature review for the past 17 years-University of Kochi Bulletin, Faculty of Social Welfare 67: 101-116.

12. Hashimoto M, Ono S (2014) Obstructive factors of EoLC in nursing home-Survey on facilities having difficulty in promotion of EoLC - Clinic of death 37(1): 142-147.

13. Shimada C, Horiuchi F, Tsuruwaka M, Takahashi R (2013) EoLC care practice in nursing homes and related factors. Japan Socio-Gerontological Society 34 (4): 500-509.

14. Fukuda Y, Takahide T, Chigusa A (2015) Consciousness for EoLC looked from the families of nursing home residents. The $33^{\text {rd }}$ Takada Junior College Bulletin, 1-12.

15. Kobayashi $G$, Hirakata $H$, Inoue $M$, Yokoyama $S$, Ishida M, et al. (2018). Study of End-of-Life Care in a Special Elderly Nursing Home. Kmj The Kitakanto Medical Journal 68(1): 31-41.

16. Kurihara A (2019) Factors influencing the choice of care for dying individuals in special nursing homes for the elderly. Elderly Care and Behavioral Science 24: 53-62.

17. Kawakami Y, Hamano J, Kotani M, Kuwata M, Yamamoto R, et al. (2019) Recognition of End-of-life Care by Nursing Care Staff, and Factors Impacting Their Recognition: An Exploratory Research Using Mixed Methods. Palliat Care Res 14(1): 43-52. 